\title{
Publisher Correction: Excess calorie intake early in life increases susceptibility to colitis
} in adulthood

Ziad AI Nabhani (iD, Sophie Dulauroy, Emelyne Lécuyer, Bernadette Polomack, Pascal Campagne, Marion Berard and Gérard Eberl (D)

Correction to: Nature Metabolism https://doi.org/10.1038/s42255-019-0129-5, published online 4 November 2019.

In the HTML version of this article initially published, the Abstract header should have read only 'Abstract' but contained additional stray text. The error has been corrected in the HTML version of the article.

Published online: 12 November 2019

https://doi.org/10.1038/s42255-019-0144-6

๑ The Author(s), under exclusive licence to Springer Nature Limited 2019 